E14-2012-44

T. N. Mamedov, A. S. Baturin ${ }^{1}$, V. N. Duginov, K. I. Gritsaj, R. Khasanov ${ }^{2}$, A. Maisuradze ${ }^{2}$, A. V. Stoykov ${ }^{2}$

\title{
RELAXATION OF THE SHALLOW ACCEPTOR CENTER IN GERMANIUM
}

Submitted to «Письма в ЖЭТФ»

\footnotetext{
${ }^{1}$ Moscow Institute of Physics and Technology, 141700 Dolgoprudny, Moscow Region, Russia

${ }^{2}$ Paul Scherrer Institut, CH-5232 Villigen PSI, Switzerland
} 
Мамедов Т. Н. и др.

Релаксация мелкого акцепторного центра в германии

Механизм релаксации мелких акцепторных центров в германии исследован с помощью поляризованных отрицательных мюонов. В германии р-типа при низких температурах обнаружена релаксация спина мюона, свидетельствующая о том, что мюонный атом, как акцепторный центр (АЦ), образуется в парамагнитном состоянии и имеет место релаксация его магнитного момента. Скорость релаксации спина мюона зависит от температуры и от концентрации примеси галлия. Полученные данные позволяют заключить, что релаксация магнитного момента акцепторного центра галлия в германии обусловлена как рассеянием фононов на АЦ, так и квадрупольным взаимодействием между акцепторами. Впервые мы оценили константу сверхтонкого взаимодействия для акцепторного центра галлия в германии как $\approx 0.11$ МГц.

Работа выполнена в Лаборатории ядерных проблем им. В.П. Джелепова ОИЯИ.

Препринт Объединенного института ядерных исследований. Дубна, 2012

Mamedov T. N. et al.

E14-2012-44

Relaxation of the Shallow Acceptor Center in Germanium

Polarized negative muons were used to study relaxation mechanisms of shallow acceptors in germanium. In p-type germanium at low temperatures relaxation of the muon spin was observed, indicating that the muonic atom (gallium-like acceptor center) formed via capture of the negative muon by a host atom is in the paramagnetic state and its magnetic moment is relaxing. The relaxation rate of the muon spin was found to depend on the temperature and on the concentration of gallium impurity. We conclude that to the relaxation of the magnetic moment of the $\mathrm{Ga}$ acceptor in Ge there contribute both scattering of phonons and quadrupole interaction between the acceptors. We estimate, for the first time, the hyperfine interaction constant for the gallium acceptor in germanium as $\approx 0.11 \mathrm{MHz}$.

The investigation has been performed at the Dzhelepov Laboratory of Nuclear Problems, JINR.

Preprint of the Joint Institute for Nuclear Research. Dubna, 2012 


\section{INTRODUCTION}

Germanium is one of two elemental semiconductors widely used in technological applications. However, the hyperfine interaction and the relaxation mechanisms of shallow acceptor centers (AC) in germanium have not been thoroughly studied so far. It is commonly assumed that in semiconductors with the diamond crystal structure the ground state of the acceptor center is four-fold degenerate, and this leads to a very high relaxation rate of the acceptor center magnetic moment due to interaction with phonons [1]. Many attempts to observe shallow acceptors in the paramagnetic state in these semiconductors by the ESR method were unsuccessful [2]. It is worth mentioning that recently superconductivity has been discovered in heavily gallium-doped germanium [3].

The probability for the gallium acceptor in germanium at thermal equilibrium to be paramagnetic is shown in Fig. 1. The calculation was done for the gallium concentration of $3 \cdot 10^{14} \mathrm{~cm}^{-3}$. As is seen, at temperature below $10 \mathrm{~K}$ the acceptors are paramagnetic, i.e., the holes are localized at the impurity atoms. As the acceptor impurity concentration increases, the onset of a decrease in the probability slightly moves to higher temperatures. The critical concentration of $\mathrm{Ga}$ atoms in germanium corresponding to the Mott transition is $1.86 \cdot 10^{17} \mathrm{~cm}^{-3}$ [5].

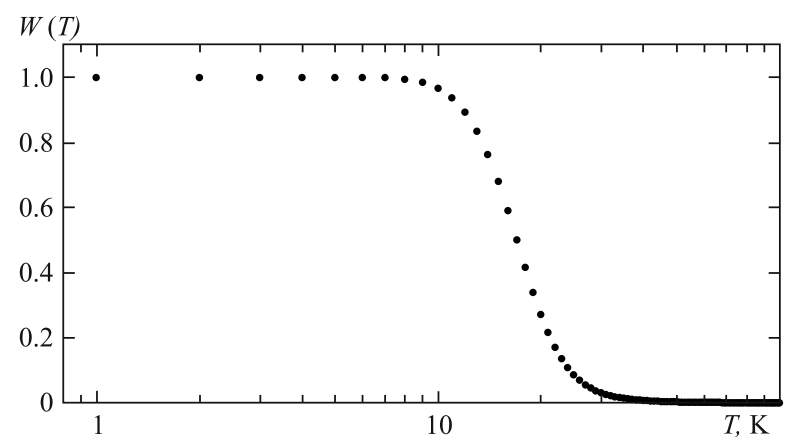

Fig. 1. Temperature dependence of the equilibrium probability for the gallium acceptor in germanium to be in the paramagnetic (non-ionized) state at Ga concentration of $3 \cdot 10^{14} \mathrm{~cm}^{-3}$. The calculation was done using the following values of the parameters [4]: energy gap $E_{\mathrm{g}}=0.66 \mathrm{eV}$, ionization energy of $\mathrm{Ga}$ acceptor $E_{\mathrm{a}}=11 \mathrm{meV}$, effective density of states in the valence band $N_{\mathrm{v}}=5.0 \cdot 10^{18} \cdot(T / 300)^{1.5} \mathrm{~cm}^{-3}$, effective density of states in the conduction band $N_{\mathrm{c}}=1.0 \cdot 10^{19} \cdot(T / 300)^{1.5} \mathrm{~cm}^{-3}$ 
Contribution to the relaxation of the magnetic moment of the acceptor center can come from its interaction with phonons and other paramagnetic centers including the neighboring acceptors and from the spin-exchange scattering of free charge carriers on the acceptor. At temperature below $10 \mathrm{~K}$ the concentration of free charge carriers is low and the contribution of the spin-exchange scattering mechanism is negligible.

As first suggested in [6], at some concentrations of acceptors in a semiconductor their quadrupole interaction can make an appreciable contribution to the relaxation of the AC magnetic moment. Within the framework of the effective mass theory it was shown that a shallow acceptor center in germanium has a nonzero quadrupole moment, and an explicit expression for the energy of quadrupole interaction between nearest-neighbor acceptors as a function of the mean distance was obtained.

In the present experiment polarized negative muons were used to study interactions of the gallium acceptor impurity in germanium. Implanted into a medium, the negative muon is slowed down and captured by a host atom. The Bohr radius of the muon in the 1 s-state is $\sim 207$ times smaller than that of the electron, and the muon effectively screens a unit of the positive charge of the nucleus. Therefore, in germanium the muonic atom imitates the gallium atom, which is known to form a shallow acceptor center. The interactions of the muonic atom with the medium, and accordingly that of the Ga acceptor, can be studied by studying the behavior of the muon-spin polarization.

\section{MEASUREMENT}

The measurements were carried out with the GPD spectrometer at the Swiss Muon Source [7] of the Paul Scherrer Institut in Switzerland.

The temperature dependence of the negative muon polarization was studied in four germanium samples: a) undoped n-type germanium with resistivity $\geqslant 45 \Omega \cdot \mathrm{cm}$ (donor concentration $\lesssim 3.0 \cdot 10^{13} \mathrm{~cm}^{-3}$ ); b) three p-type samples with Ga concentrations $1.0 \cdot 10^{14} \mathrm{~cm}^{-3}$ (resistivity $\approx 30 \Omega \cdot \mathrm{cm}$ ), $3.0 \cdot 10^{14} \mathrm{~cm}^{-3}$, and $5.0 \cdot 10^{15} \mathrm{~cm}^{-3}$. The $\mu \mathrm{SR}$ measurements were carried out in the transverse to the muon spin magnetic field $H=2.5 \mathrm{kOe}$. The Oxford-He 3 cryostat, used in this experiment, allowed us to cool the samples down to $0.23 \mathrm{~K}$.

The $\mu \mathrm{SR}$ spectra (time distribution of electrons from the $\mu^{-} \rightarrow e^{-}$decay) were fitted by the following function:

$$
\begin{aligned}
N(t)= & N_{0} \exp \left(-t / \tau_{\mu}\right)[1+a P(t)]+\mathrm{Bg}, \\
& P(t)=P_{0} \exp (-\lambda t) \cos (\omega t+\varphi),
\end{aligned}
$$

where $N_{0}$ is proportional to the number of muons stopped in the sample; $\tau_{\mu}$ is the life time of the negative muon in the 1s-state in the germanium atom; $a$ is the 
asymmetry coefficient of the space distribution of the decay electrons; $P(t)$ is the projection of the muon polarization onto the direction to the electron telescope; $P_{0}$ is the muon polarization in the 1 s-state at $t=0 ; \lambda$ is the relaxation rate of the muon spin polarization; $\omega$ and $\varphi$ are the frequency and the initial phase of the muon spin precession in the magnetic field; $\mathrm{Bg}$ is the uncorrelated background.

The muon life time in the $1 \mathrm{~s}$-state in germanium is $166.5 \mathrm{~ns}$ [8]. This means that about $92 \%$ of muons disappear captured by the nucleus, and only $8 \%$ of them decay with emission of an electron. Accordingly, the data acquisition rate in this experiment is rather low.

\section{RESULTS AND DISCUSSION}

The time dependence of the muon-spin polarization in the n-type Ge sample at $6 \mathrm{~K}$ is shown in Fig. 2. Analogous time spectra were also observed at temperatures $0.3,3.1,10$, and $15 \mathrm{~K}$. The Fourier analysis of the $\mu$ SR histograms (see Fig. 3) reveals a single peak at the frequency corresponding to the precession of the free muon spin in the applied external magnetic field. The relaxation of the muon spin is not observed. This result evidences that, unlike the case in n-type silicon [9], in n-type germanium the muonic atom forms in the diamagnetic state within the time $\lesssim 10^{-9}$ s and the concentration of non-equilibrium charge carriers (produced during the muon slowing-down and formation of the muonic atom) is negligible at times $\geqslant 10^{-9} \mathrm{~s}$

In contrast to n-type germanium, in p-type samples at low temperatures relaxation of the muon spin was observed (see Fig.4). For the samples with Ga concentrations $\approx 1.0 \cdot 10^{14} \mathrm{~cm}^{-3}$ and $\approx 3.0 \cdot 10^{14} \mathrm{~cm}^{-3}$ the temperature dependences of the muon spin relaxation rate are similar. At the Ga concentration of $\approx 5.0 \cdot 10^{15} \mathrm{~cm}^{-3}$ the relaxation rate is smaller and its temperature dependence is different. The drop of the muon spin relaxation rate at temperatures higher

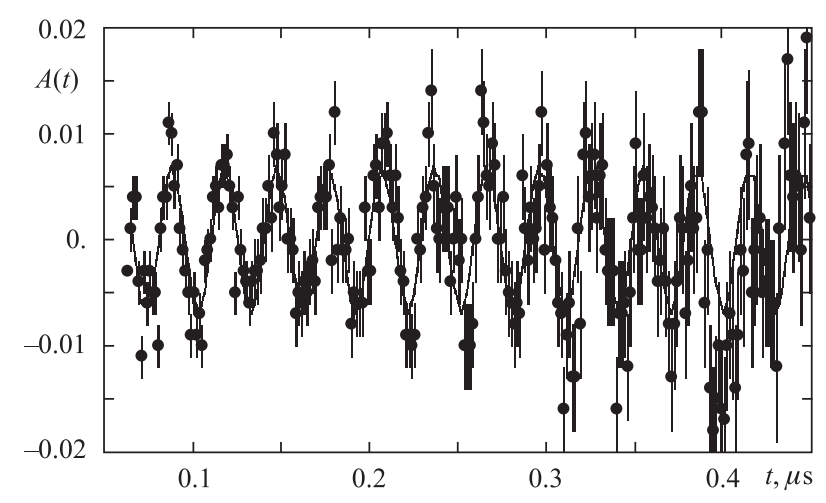

Fig. 2. Negative muon spin polarization $A(t)=a P(t)$ in the n-type Ge sample at $6 \mathrm{~K}$ 


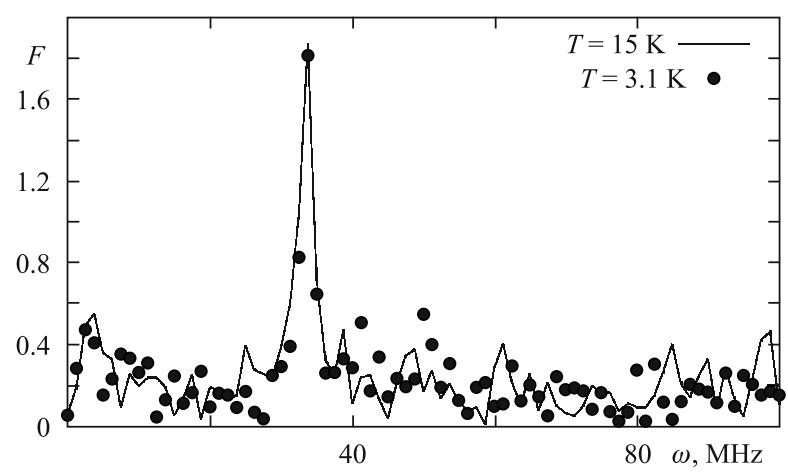

Fig. 3. Fourier analysis of the $\mu \mathrm{SR}$ spectra in the n-type Ge sample at $3.1 \mathrm{~K}$ and $15 \mathrm{~K}$

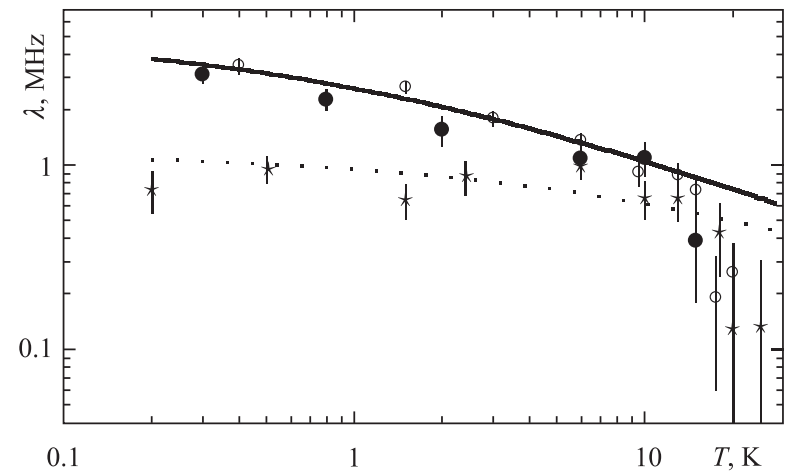

Fig. 4. Temperature dependence of the relaxation rate of the negative muon spin in p-type Ge: closed circles correspond to the Ga concentration $1.0 \cdot 10^{14} \mathrm{~cm}^{-3}$; opened circles, to $3 \cdot 10^{14} \mathrm{~cm}^{-3}$; and crosses, to $5 \cdot 10^{15} \mathrm{~cm}^{-3}$. The solid and dotted lines correspond to approximation of the function (2) to the experimental data (see the text)

than $12 \mathrm{~K}$ corresponds to ionization of the muonic atom as expected for the Gaacceptor in germanium (see Fig. 1). Above $30 \mathrm{~K}$ no relaxation is observed in any sample.

According to the theoretical calculation [10], if a muonic atom as an acceptor center is formed in the paramagnetic state, the relaxation of the negative muon spin is conditioned by the hyperfine interaction between the muon spin and the magnetic moment of the acceptor center. The relaxation rate of the muon spin depends on that of the acceptor center magnetic moment as

$$
\lambda=\frac{J(J+1)}{3} \frac{(A / \hbar)^{2}}{\nu}\left(1+\frac{1}{1+\left(\omega_{\mathrm{e}} / \nu\right)^{2}}\right),
$$


where $J=3 / 2$ is the angular momentum of the Ga acceptor center in germanium, $A$ is the hyperfine interaction constant, $\nu$ is the relaxation rate of the AC magnetic moment, $\hbar=h / 2 \pi, h$ is the Planck constant, $\omega_{\mathrm{e}}=g \mu_{\mathrm{B}} B / \hbar$ is the angular precession frequency of the AC magnetic moment in the magnetic field $B, \mu_{\mathrm{B}}$ is the Bohr magneton, and $g$ is the g-factor of the AC.

Therefore, our experimental data evidence that the relaxation rate of the magnetic moment of the Ga acceptor in germanium increases with increasing impurity concentration, and at the concentration of $5.0 \cdot 10^{15} \mathrm{~cm}^{-3}$ it practically does not depend on temperature in the range of $0.3-10 \mathrm{~K}$. It is worth mentioning that earlier analogous behavior of the relaxation time $\mathcal{T}_{2}$ of the Ga acceptor in germanium was observed in an ultrasonic absorption experiment [6] (Ga concentration $3.5 \cdot 10^{15} \mathrm{~cm}^{-3}$, temperature range $1.4-4.2 \mathrm{~K}$ ). The effect was explained by the electric quadrupole interaction between the acceptor centers.

According to [11], the mean distance between randomly distributed acceptors with concentration $N=5.0 \cdot 10^{15} \mathrm{~cm}^{-3}$ is $d=(4 \pi N / 3)^{-1 / 3} \Gamma(4 / 3) \approx$ $0.5539 N^{-1 / 3}=324 \AA$. This is about 9 times larger than the effective Bohr radius $a^{*}=35 \AA$ of the $\mathrm{Ga}$ acceptor center in germanium [12]. Therefore we can disregard the contribution of the spin-exchange interaction between acceptor centers to the relaxation of the acceptor center magnetic moment at Ga concentrations $\leqslant 5.0 \cdot 10^{15} \mathrm{~cm}^{-3}$.

The experimental results can be interpreted in view of the contribution of two mechanisms for the relaxation of the AC magnetic moment: 1) interaction of AC with phonons, and 2) quadrupole interactions between AC. As is known, in nondegenerate semiconductors the interaction with phonons depends on temperature and does not depend on the impurity concentration. One-phonon and Raman processes lead to the power-low temperature dependence of the relaxation rate: $\nu_{\mathrm{ph}} \sim T^{\alpha}[6,13,14]$. At the same time, the quadrupole interaction depends on the concentration of acceptor centers. Therefore, here we assume that the relaxation rate of the $\mathrm{AC}$ magnetic moment depends on temperature as

$$
\nu=\nu_{\mathrm{ph}}+\nu_{\mathrm{q}}=\operatorname{const}\left(\left(T / T_{0}\right)^{\alpha}+b\right),
$$

where $T_{0}=1 \mathrm{~K}$. The term $b$ is due to the quadrupole interaction and is equal to the ratio of the quadrupole to the phonon relaxation rate at $1 \mathrm{~K}$.

The experimental data on temperature dependence of $\lambda$ at $T \lesssim 13 \mathrm{~K}$ were fitted by the function $\lambda(T)=C / \nu$ (we disregarded the second term in equation (2), taking into account that $\omega_{\mathrm{e}} / \nu>10$ ). It was found that $\alpha=0.84 \pm 0.17$, $b=2.2 \pm 1.3$ for the samples with $\mathrm{Ga}$ concentration $1.0 \cdot 10^{14} \mathrm{~cm}^{-3}$ and $3.0 \cdot 10^{14} \mathrm{~cm}^{-3}$, and $b=8.6 \pm 0.8$ at the $\mathrm{Ga}$ concentration $5.0 \cdot 10^{15} \mathrm{~cm}^{-3}$. The dominant role of the quadrupole interaction between the neighboring acceptors in the relaxation of the magnetic moment of the Ga acceptor impurity in germanium at the $\mathrm{Ga}$ concentration of $5.0 \cdot 10^{15} \mathrm{~cm}^{-3}$ is obvious. 
As follows from our experimental data, the contribution of the phonon processes to the relaxation of $\mathrm{AC}$ in germanium is proportional to $T^{0.84}$. This is close to that predicted theoretically in the high-temperature limit in the absence of external magnetic fields for the single-phonon process: $\nu_{\mathrm{ph}} \sim T$ [6]. For the Raman process one can expect, similar to the case of silicon, a much stronger temperature dependence of the relaxation rate: $\nu_{\mathrm{ph}} \sim T^{\alpha}, 3 \lesssim \alpha \lesssim 5[9,13,14]$.

Using the present experimental data on the muon spin relaxation rate in the sample with the Ga concentration of $5.0 \cdot 10^{15} \mathrm{~cm}^{-3}$, the analytical relation (2), and the results of [6] on the relaxation time $\mathcal{T}_{2}\left(\nu=1 / \mathcal{T}_{2}=1.25 \cdot 10^{8} \mathrm{~s}^{-1}\right)$, we estimate the hyperfine interaction constant for the muonic atom in germanium to be $A_{\mathrm{hf}}\left({ }_{\mu} \mathrm{Ga}\right) \approx 1.5 \mathrm{MHz}$. Taking into account the ratio between the magnetic moments $\mu_{\mu} / \mu_{\mathrm{N}}$ and the spins $S_{\mu} / I$ of the muon and the nucleus of Ga, and the relation $A_{\mathrm{hf}}(\mathrm{Ga}) / A_{\mathrm{hf}}\left({ }_{\mu} \mathrm{Ga}\right)=\left(\mu_{\mathrm{N}} / \mu_{\mu}\right)\left(S_{\mu} / I\right)$, we estimate for the first time the hyperfine interaction constant for the gallium acceptor in germanium as $A_{\text {hf }}(\mathrm{Ga}) \rightleftharpoons 0.11 \mathrm{MHz}$.

In summary, it is shown that at the concentration of gallium impurity in germanium $5.0 \cdot 10^{15} \mathrm{~cm}^{-3}$ the quadrupole interaction between the acceptor centers plays the dominant role in the relaxation of the acceptor center magnetic moment. For the first time the hyperfine interaction constant for the gallium acceptor center in germanium is estimated as $\approx 0.11 \mathrm{MHz}$.

We are grateful to the Directorate of the Paul Scherrer Institut for the possibility of carrying out this experiment.

\section{REFERENCES}

1. Bir G. L., Pikus G. E. Symmetry and Strain-Induced Effects in Semiconductors. N. Y.: Wiley, 1975.

2. Watkins G. D. // Phys. Solid State. 1999. V.41. P. 746.

3. Herrmannsdörfer T., Skrotzki R., Heeraet V. et al. // Supercond. Sci. Technol. 2010. V.23. P. 034007.

4. http://www.ioffe.rssi.ru/SVA/NSM/Semicond/Ge/index.html (06.04.2012)

5. Itoh K. M., Haller E. E., Beemanet J. W. et al. // Phys. Rev. Lett. 1996. V.77. P. 4058.

6. Tokumoto H., Ishiguro T., Kajimura K. // J. Phys. C: Solid St. Phys. 1980. V. 13. P. 4061.

7. http://lmu.web.psi.ch/facilities/gpd/gpd.html (06.04.2012)

8. Suzuki T., Measday D. F., Roalsvig J. P. // Phys. Rev. C. 1987. V.35. P. 2212.

9. Mamedov T. N., Gorelkin V.N., Stoykov A. V. // Phys. Part. Nucl. 2002. V.33. P. 519.

10. Gorelkin V. N., Mamedov T. N., Baturin A. S. // Physica B. 2000. V. 289-290. P. 585.

11. Chandrasekhar S. // Rev. Mod. Phys. 1943. V.15. P.1.

12. Eizo Otsuka et al. // J. Phys. Soc. Japan. 1966. V.21. P. 1104.

13. Shimizu T., Nakayama M. // J. Phys. Soc. Japan. 1964. V. 19 (10). P. 829.

14. Yafet Y. // J. Phys. Chem. Solids. 1965. V. 26. P. 647.

Received on April 19, 2012. 


\section{Корректор T. Е. Попеко}

Подписано в печать 7.06.2012.

Формат $60 \times 90 / 16$. Бумага офсетная. Печать офсетная.

Усл. печ. л. 0,68. Уч.-изд. л. 0,92. Тираж 250 экз. Заказ № 57671.

Издательский отдел Объединенного института ядерных исследований 141980, г. Дубна, Московская обл., ул. Жолио-Кюри, 6.

E-mail: publish@jinr.ru www.jinr.ru/publish/ 